EGU2020-2919

https://doi.org/10.5194/egusphere-egu2020-2919

EGU General Assembly 2020

(c) Author(s) 2021. This work is distributed under

the Creative Commons Attribution 4.0 License.

\title{
A global bibliometric perspective on soil erosion modelling
}

\author{
Nejc Bezak ${ }^{1}$ and the Soil Erosion Modelling Team ${ }^{*}$ \\ ${ }^{1}$ University of Ljubljana, Faculty of civil and geodetic engineering, Ljubljana, Slovenia (nejc.bezak@fgg.uni-lj.si) \\ ${ }^{*}$ A full list of authors appears at the end of the abstract
}

Systematic bibliometric investigations are useful to evaluate and compare the scientific impact of journal papers, book chapters and conference proceedings. Such studies allow the detection of emerging research topics, the analyses of cooperation networks, and the collection of in-depth insights into a specific research topic. In the presented work, we carried out a bibliometric study in order to obtain an in-depth knowledge on soil erosion modelling applications worldwide.

As a starting point, we used the soil erosion modelling meta-analysis data collection generated by the authors of this abstract in a joint community effort. This database contains meta-information of more than 3,000 documents published between 1994 and 2018 that are indexed in the SCOPUS database. The documents were reviewed and database entries verified. The database contains various types of meta-information about the modelling studies (e.g., model used, study area, input data, calibration, etc.). The bibliometric information was also included in the database (e.g., number of citations, type of publication, Scopus category, etc.). We investigated differences among publication types and differences between papers published in journals that are part of various Scopus categories. Moreover, relationships between publication CiteScore, number of authors, and number of citations were analyzed. A boosted regression tree model was used to detect the relative impact of the selected meta-information such as erosion model used, spatial modelling scale, study period, field activity on the total number of citations. Detailed investigation of the most cited papers was also conducted. The VOSviewer software was used to analyze citations, cocitations, bibliographic coupling, and co-authorship networks of the database entries.

Our bibliometric investigations demonstrated that journal publications, on average, receive more citations than book series or conference proceedings. There were differences among the erosion models used, and some specific models such as the WaTEM/SEDEM model, on average, receive more citations than other models (e.g., USLE). It should also be noted that self-citation rates in case of most frequently used models were similar. Global studies, on average, receive more citations than studies dealing with plot, regional, or national scales. According to the boosted regression tree model, model calibration, validation, or field activity do not have significant impact on the obtained publication citations. Co-citation investigation revealed some interesting patterns. Our results also indicate that papers about soil erosion modeling also attract citations from different fields and better international cooperation is needed to advance this field of research with regard to its visibility and impact on human societies. 
Soil Erosion Modelling Team: Pasquale Borrelli, Christine Alewell, Pablo Armando Alvarez Figueroa, Jamil Alexandre Ayach Anache, Jantiene Baartman, Cristiano Ballabio, Marcella Biddoccu, Artemi Cerda, Devraj Chalise, Changjia Li, Songchao Chen, Walter Chen, Anna Maria De Girolamo, Gizaw Desta, Detlef Deumlich, Nikolaos Efthymiou, Gunay Erpul, Peter Fiener, Michele Freppaz, Francesco Gentile, Andreas Gericke, Nigussie Haregeweyn, Bifeng Hu, Amelie Jeanneau, Konstantinos Kaffas, Mahboobeh Kiani-Harchegani, Ivan Lizaga Villuendas, Luigi Lombardo, Manuel López-Vicente, Manuel Esteban Lucas-Borja, Michael Märker, Chiyuan Miao, Matjaž Mikoš, Markus Möller, Victoria Naipal, Mark Nearing, Stephen Owusu, Panos Panagos, Dinesh Panday, Edouard Patault, Cristian Patriche, Laura Poggio, Raquel Portes, Laura Quijano Gaudes, Mohammad Reza Rahdari, Mohammed Renima, Giovanni Francesco Ricci, Jesús Rodrigo-Comino, Sergio Saia, Aliakbar Nazari Samani, Calogero Schillaci, Kim Hyuck Soo, Diogo Noses Spinola, Paulo Tarso Oliveira, Hongfen Teng, Resham Thapa, Konstantinos Vantas, Diana Vieira, Jae E. Yang, Shuiqing Yin, Demetrio Antonio Zema, Guangju Zhao 